\title{
Políticas de formación para mujeres inmigrantes. El caso de la ciudad de Barcelona
}

\author{
Amado Alarcón; Francesc Gibert; Sònia Parella \\ Natalia Ribas (coord.) \\ Universitat Autònoma de Barcelona. Departament de Sociologia i Ciències Polítiques \\ Centre d'Estudis d'Immigració i Minories Ètniques \\ 08193 Bellaterra (Barcelona). Spain
}

\section{Resumen}

El artículo expone los resultados de un trabajo centrado en el análisis de las políticas de formación dirigidas a las mujeres inmigrantes extranjeras en un contexto local preciso, la ciudad de Barcelona. El estudio presenta el material resultante de un taller de investigación en el marco de una asignatura de doctorado de Sociología (Universitat Autònoma de Barcelona). Aparte del material descriptivo sobre los programas existentes, se han utilizado técnicas cualitativas basadas en entrevistas en profundidad a "entrevistados privilegiados». El análisis se ha organizado a partir de tres bloques básicos: la filosofía subyacente en dichas políticas, la aplicación de un modelo de interacción entre los diferentes actores sociales (institucionales, cuasi-institucionales y extra-institucionales) en un ámbito de welfare mix, y la contextualización de dichas políticas en las dinámicas del mercado de trabajo.

Palabras clave políticas de formación, ciudad de Barcelona, actores sociales (institucionales, cuasi-institucionales y extra-institucionales), welfare mix.

\section{Abstract Training policies for mig rant women. The case of $\mathrm{Ba}$ rcelona}

This article shows the result of a research on the analysis of training policies addressed to foreign migrant women in the city of Barcelona. This work is a result of workshop during a PhD Sociology course (University Autònoma de Barcelona). Apart from the descriptive material on the existing programmes, qualitative techniques focused on in-depth interviews to key-informants have been adopted. The analysis has been organised according to three thematic topics: the philosophy behind those policies, the adoption of an interaction model among the different social actors (institutional, cuasi-institutional and extra-institutional) in a welfare mix context and the contextualisation of those policies in the labour market dynamics.

Key wods: training policies, city of Barcelona, social actors (institutional, cuasi-institutional and extra-institutional), welfare mix.

\section{Sumario}

\section{Introducción}

2. Realización de entrevistas

3. Del mercado a las políticas

4. El doble desdibujamiento de la gestión: modelo horizontal y vertical
5. El análisis de la interacción definido por el welfare mix 6. El resultado de las políticas: una solución con dos caras Bibliografía 


\section{Introducción}

El contexto de la ciudad y la capacidad de respuesta de las políticas urbanas en relación con la inmigración se presenta como uno de los temas clave de las metrópolis europeas en el final del milenio. En este sentido, el papel que tiene la ciudad de Barcelona ha sido considerado frecuentemente un interesante modelo de análisis, pese a destacarse por su inexperiencia, como el resto de la Europa mediterránea. Ello se debe no sólo a su antigua experiencia asociada a las migraciones interiores de los años sesenta, sino también al carácter innovador que han presentado sus políticas de integración social de los inmigrantes extranjeros en comparación con el resto de las ciudades del Estado español e incluso, a veces, en comparación con otras ciudades del sur de Europa.

El interés de este trabajo radica en situar las dinámicas de una de estas políticas en el contexto de la ciudad: la formación de las mujeres inmigrantes. Para ello, se analizará la evaluación y coordinación de dicha casi invisible política social, con la finalidad de esbozar su funcionamiento y su incidencia en el caso de Barcelona. Desde el ámbito institucional nos referimos a las diferentes administraciones (central, autonómica y local —ayuntamiento y diputación-); desde el ámbito del tercer sector nos centramos en el tipo de modelo de interacción que se establece entre el tejido social y la política formativa para mujeres inmigrantes. Desde el punto de vista institucional, las interacciones entre actores van mucho más allá del ámbito local y autonómico, puesto que el marco legislativo en materia de formación y de inmigrantes no sólo está configurado por el marco nacional, sino también por las estructuras de subvenciones que traspasan los límites nacionales y se sitúan en el nivel de las instituciones europeas.

En sentido amplio, las políticas de empleo y del mercado de trabajo intentan suplir las desigualdades presentes en el mercado a partir de la heterogeneidad de los diferentes grupos que participan en el mercado. Aquí nos referimos no a las políticas de regulación laboral, sino a aquéllas que tratan de determinados aspectos del mercado. Entre las políticas activas de empleo, que son las que a nosotros nos incumben, podemos distinguir, según Sáez (1997: 310): 1) aquéllas que persiguen desarrollar un buen sistema de intermediación entre la oferta y la demanda a través de medidas de diversa naturaleza; 2) las actuaciones destinadas a mejorar los mecanismos de educación y formación a efectos de facilitar a los jóvenes una base de partida que les permita desempeñar una profesión y avanzar en la cualificación profesional de los adultos, y 3) las que tienen que ver con la promoción del empleo. A nosotros nos interesa especialmente ver la combinación de medidas con relación a la orientación, formación y experiencia laboral. Respecto a los efectos de tales políticas nos fijaremos en la operatividad de los programas, porque tendrán efectos distintos según a la población-objetivo a la que se refieran, así como en el contexto en el que se apliquen.

En los países industrializados se ha registrado en la última década un incremento de las políticas dirigidas a los colectivos con baja tasa de empleabilidad. 
Según Sáez el grupo más importante ha sido el de los desempleados de larga duración (superior a un año) y que superan cierta edad, el segundo grupo sería aquéllos cuya experiencia laboral es muy pobre o inexistente (marginación, historial delictivo, inmigración, baja cualificación) y el tercer grupo serían los jóvenes recientemente incorporados al mercado de trabajo. Otros grupos de segunda línea son los considerados como inactivos (especialmente se refieren a las mujeres) y los receptores de rentas sociales, a los que se empuja hacia el empleo como mecanismo de integración social.

Además de realizar una labor descriptiva, en la que se fotografían programas y se traducen cifras, el propósito general de este artículo es buscar una primera aproximación a través de la cual analizar la interrelación de actores en el diseño de las políticas relacionadas con la formación de mujeres inmigrantes, tanto a partir de la relación entre los diferentes actores con las administraciones, como desde el análisis completo de cada actor. Dicho análisis se realizará a partir del examen de tres elementos: la ideología, el sistema de relaciones y el papel que tiene cada actor (el tipo de servicio que presta). A nivel institucional, vemos que, a pesar de que nuestro interés se centre en el ámbito local, el análisis supera dicha delimitación de lo local. Así pues, es necesario también recabar en el sistema político general y examinar los mecanismos que se están aplicando respecto a los temas de extranjería. Es decir, se trata de observar la política de inmigración, sobre todo la de integración social, como resultado de una cultura política concreta. Este fenómeno se refleja por ejemplo en las experiencias europeas donde las políticas de inmigración funcionan como «efecto reflejo» o incluso como un «efecto amplificador» de los modelos políticos instaurados a nivel nacional (ver Caponio y otros, en este monográfico).

Se ha intentado partir de un enfoque dinámico que incluya la interacción de todos los actores sociales escogidos con el fin de recoger el proceso de negociación con relación a las políticas públicas dirigidas a la formación de mujeres inmigrantes en la ciudad de Barcelona. El análisis recoge una evolución concreta del tema en relación a la migración femenina, el cual se inició, aproximadamente, a partir de la I Conferencia de Mujeres Inmigrantes que tuvo lugar en Barcelona en 1994.

\section{Realización de entrevistas}

No se trata aquí de ver cómo funcionan las políticas de emergencia para poblaciones recién llegadas, sino que concebimos la formación como un elemento de las políticas de asentamiento respecto a la población inmigrante. En este sentido, nos adelantamos un poco en el tiempo y suponemos que una primera fase de «acogida» ha sido finalizada. Por otra parte, se ha seleccionado el tema de la mujer inmigrante como "población objetivo" de estas políticas, no sólo por la peculiar situación en el mercado de trabajo, sino porque además la construcción de estereotipos desde los diferentes actores sociales hace bastante generalizable la imagen de las mujeres inmigrantes como una población "necesitada" por excelencia. Discriminadas y explotadas en sus lugares de origen, también 
discriminadas y explotadas en el mundo rico donde esperan mejorar sus condiciones de vida, las mujeres pobres son vistas como "pobres mujeres» a las que se puede utilizar. Con una perspectiva más progresista, se piensa que hay que ayudarlas y educarlas (Juliano, 1998: 70).

Hemos utilizado la entrevista cualitativa a «informantes privilegiados» como técnica de recogida de datos. Para ello, se ha confeccionado un guión de entrevista más o menos común, que ha sido adaptado en función de las características de cada uno de los actores implicados. Se ha entrevistado a representantes de la Administración en el ámbito autonómico y local y a responsables de formación que fueran lo máximamente representativos de los actores cuasi-institucionales y de los extra-institucionales que participan directamente en la acción formativa dirigida a mujeres inmigrantes. En total se llevaron a cabo sólo siete entrevistas, las cuales presentamos a continuación:

- Por lo que se refiere a los actores institucionales, se han realizado cuatro entrevistas: a un representante de la Subdirecció de Col-lectius amb Especials Dificultats del Departament de Treball de la Generalitat de Catalunya; a la directora del órgano técnico del Pla Interdepartamental d'Immigració de la Generalitat de Catalunya; a la coordinadora de los programas de formación para inmigrantes de la Diputació de Barcelona y, por último, a un representante del Comissionat de Drets Civils de l'Ajuntament de Barcelona.

- Por lo que se refiere a los actores cuasi-institucionales, tres han sido las entrevistas efectuadas, dos de ellas a los sindicatos más representativos en materia de formación y otra a una institución fuertemente vinculada a la Iglesia Católica (Cáritas). Se ha entrevistado a la responsable de formación de IDFO, entidad de formación ocupacional y continua de la UGT; al responsable de planificación del CITE, entidad de asesoramiento de los trabajadores extranjeros de la Comissió Obrera Nacional de Catalunya (CONC) y a la coordinadora pedagógica del Servei d'Atur de Cáritas.

- Finalmente, los actores extra-institucionales escogidos han sido dos: la directora de una asociación de mujeres, SURT (Associació de Dones per a la Inserció Laboral) y la responsable del Centro Peruano de Barcelona.

Lógicamente, la selección de entrevistados es poco exhaustiva y los distintos actores no están suficientemente representados en una muestra tan reducida. Una de las carencias principales de la muestra es el hecho de dejar de lado a la población objeto de estudio, en tanto que no hemos entrevistado a ninguna mujer inmigrante que pueda contarnos en primera persona cuáles son sus «necesidades» $y$ demandas de formación. Contamos pues con un sesgo metodológico de partida con la consecuente pérdida de información que ello conlleva. La principal causa de este «vacío» es la limitación de recursos con la que hemos contado. Hemos intentado solventar este problema a través del discurso que ofrecen las asociaciones, puesto que es el tejido asociativo el que mejor canaliza las demandas de este colectivo. No obstante, sólo dos entrevistas recogen la perspectiva de los actores extra-institucionales, de modo que el tejido asociativo no se cubre con detenimiento y se dejan de lado asociaciones de 
inmigrantes con una fuerte tradición en materia formativa (Bayt al Thaqafa o el Centro Filipino, por ejemplo). La asociación SURT ha sido seleccionada por su importante tradición como centro de inserción laboral de mujeres y, en especial, de mujeres magrebíes y subsaharianas. Además, se trata de un centro fuertemente vinculado con algunas de las asociaciones de mujeres inmigrantes de la ciudad (especialmente gambianas y marroquíes), con lo que garantizamos, en cierta medida, su representatividad. Se optó también por entrevistar al Centro Peruano de Barcelona, dado que permite conocer la situación específica del colectivo de mujeres inmigrantes procedentes de los nuevos flujos de inmigración latinoamericana, dado que sus necesidades formativas son muy distintas a las que intenta hacer frente la mencionada asociación SURT.

Respecto a la eficacia de los programas formativos, ésta se mide cuantitativamente en términos de la tasa de inserción laboral. No obstante, por otra parte, dicha eficacia topa con un ámbito más amplio de integración social, en el que se activan determinados estereotipos sobre la población objetivo y una determinada concepción etnocéntrica sobre cómo se debe tratar y formar a dicha población para que se produzca la integración social en la sociedad receptora. Además, el diseño de algunos de los cursos se realiza en función de las dificultades lingüísticas y las especificidades étnicas, lo cual tiende a reproducir las diferencias entre poblaciones que percibe la sociedad en general. De ese modo, los estereotipos en el diseño de las políticas y en la gestión de las soluciones, en muchos casos, no se distinguen del discurso que mantiene la población general.

\section{Del mercado a las políticas}

Posiblemente, antes de analizar qué formación se quiere dar a un colectivo determinado al que se le asigna doblemente una situación de desventaja en el mercado de trabajo - por su condición de inmigrante extranjero y por su condición de mujer- deberíamos primeramente ver qué sucede con dicho colectivo en el mercado, para ver si los programas propuestos pueden realmente operar en un contexto real o si, por el contrario, es una previsión de cambio totalmente utópica.

Las políticas de inmigración pueden distinguirse según su función: por una parte, las políticas de control que canalizan los flujos de entrada regular de la población extranjera y, por otra, las políticas sociales, las comúnmente denominadas "políticas de integración de los inmigrantes», donde se insertarían las de formación.

Mientras que la Ley de Extranjería se centra en las políticas de entrada restrictiva, con los años hemos visto que las políticas de control del norte del Mediterráneo como guardián de Europa ha acabado abriendo cuestiones claves sobre el funcionamiento del Estado del bienestar. Así, durante los años noventa, la cuestión de la integración social de los inmigrantes y de la interculturalidad en las políticas sociales ha ido más allá de los ámbitos clásicos de 
intervención social diferenciada, como son la educación y la sanidad. La nueva Ley de Extranjería incluye pues muchos de esos ámbitos de derechos sociales anteriormente olvidados: acceder a una oferta pública de empleo, recibir ayudas para comprarse una vivienda de protección oficial, disfrutar de las prestaciones de seguridad social e incluso votar en las elecciones municipales. En relación con las respuestas de formación para mujeres inmigrantes, trataremos de ver si podemos encontrar indicios de unas líneas de formación específicas para éstas o no.

El servicio doméstico, sobre todo en su modalidad de interna, ocupa a una gran parte de la población femenina extranjera en países del sur de Europa como España, Italia y Grecia. La existencia de estos flujos se explica a partir de múltiples cambios relacionados con la proletarización femenina en las migraciones internacionales de trabajo (Ribas, 1998), desvelando que tanto en el país de origen como en el país de destino son claves los sistemas de producción-reproducción. En este respecto, siguiendo las tesis de Walby (1986), el patriarcado y el capitalismo presentan una cierta imbricación y funcionalidad. El proceso de acumulación de capital requiere de la dependencia del colectivo femenino respecto al masculino, pero, mientras que la lógica de la mercantilización de la fuerza de trabajo también comporta en muchas ocasiones la inserción del mercado de trabajo de mano de obra femenina, la lógica del patriarcado se traduce en preferencias que tienen como elemento común el relegar las mujeres a la esfera de la reproducción al hogar/familia. Esta imbricación entre patriarcado y capitalismo, además del legado colonial reproductor de las antiguas dominaciones entre países, explica en buena parte el contexto de donde emergen las estrategias de las migrantes internacionales.

En cuanto a la explicación de la feminización de los flujos desde los países de recepción, podemos ver cómo en el caso español el rol estatal ha tenido un papel importante a la hora de configurar una dinámica migratoria específica, a través del fomento de los flujos de inmigración feminizados desde el sistema de cuotas (desde 1993), puestos de trabajo cubiertos en gran parte por el servicio doméstico. Esta política de inmigración es la respuesta del EstadoNación a una prospectiva de demanda laboral que siempre ha supuesto que dicha ocupación es «un trabajo de mujeres». El análisis de esta demanda femenina se inserta a su vez en el crecimiento de los servicios en las economías de las sociedades posindustriales. Esta demanda debe comprenderse en el desarrollo de tres tipos de dinámicas que coinciden con tres áreas determinadas de las economías desarrolladas: salud, servicio doméstico y ocio, sectores también clásicos de la incorporación de la fuerza de trabajo femenina.

En España, ambos, el Estado y el mercado son coprotagonistas de la feminización de los flujos. El Estado, a través de la política de inmigración articulada por las prácticas institucionales que fijan la entrada y la inserción de los flujos en función de la gestión de cupos migratorios. El mercado, a través de una estructura de la demanda en función de las necesidades de las mujeres empleadoras del servicio doméstico, pertenecientes a las clases medias urbanas. Estas mujeres autóctonas utilizan formas mercantilistas de reproducción posible- 
mente ante la ausencia de políticas familiares que les faciliten el cuidado de los hogares; en este sentido se vuelve a reproducir de nuevo el vínculo entre un mercado muy segmentado y un débil régimen de bienestar. Ante dichos vacíos que aseguren los cuidados cotidianos, las mujeres autóctonas miran hacia la inmigración internacional para resolver las ausencias en la esfera reproductiva (Oso, 1998). Además, el hecho de que esto suceda especialmente en el contexto de las grandes ciudades hace que el contexto urbano sea muy interesante para poner en marcha los estudios de caso.

La doble vertiente de las políticas desde el Estado y el mercado puede verse claramente a partir, por un lado, de los cambios recientes relativos a las políticas de reordenación del tiempo de trabajo en los procesos productivos y, por el otro, en la dinámica del ejército de trabajo industrial en las ocupaciones femeninas en el sector secundario del mercado de trabajo. En primer lugar, estos cambios se insertan en el eje de los enfoques producción-reproducción, a partir del dilema mercantilización-desmercantilización del trabajo doméstico o de la tríada acumulación de capital-relaciones de clases-trabajo de reproducción, subordinando la cuestión de la división sexual del trabajo como un marco de variables constante. En segundo lugar, las relaciones de producción y reproducción vienen determinadas especialmente, o de forma inmediata, por las características de la división del trabajo en el modo de producción capitalista y las relaciones sociales de producción que estructuran los procesos de trabajo. En tercer lugar, también deben tenerse presente las provisiones públicas-estatales que inciden en los procesos de mercantilización y de desmercantilización de la fuerza de trabajo, como son las características de prestaciones por desempleo, las prestaciones por jubilación, así como un marco amplio en el que se insertan las políticas familiares. Así pues, una lectura transversal de la producción/reproducción requiere de un análisis de la producción de bienes y servicios, así como las dinámicas en que esta esfera incide y se ve condicionada por la "autonomía relativa» de los hogares/familias. A partir de los cambios en esta lectura de producción/reproducción se atisba el rol de los «servicios de proximidad» como nuevos yacimientos de ocupación. Este protagonismo de los «servicios de proximidad» se comprende a partir de la continua expulsión de mano de obra del sector industrial, de la creciente desregulación estatal de servicios y prestaciones asociaciadas con la reproducción de la fuerza de trabajo, el envejecimiento de la población, la modificación de las estructuras familiares, etc.

Cuando hablamos de inserción laboral de las mujeres inmigrantes en el sector secundario más feminizado y más desprestigiado socialmente de la economía, vemos cómo esta inserción se materializa en las actividades «típicamente» femeninas; es decir, se fomenta una formación dirigida hacia los «nuevos yacimientos de empleo» vinculados a los «servicios de proximidad» ${ }^{1}$ (servicios

1. Servicios destinados a satisfacer las necesidades de las personas y de las familias que aparecen en la vida cotidiana de las sociedades occidentales (Torns, 1995b: 7). 
de limpieza, cocina, cuidado de ancianos...) y, frecuentemente, prescinde de la trayectoria laboral previa de las inmigrantes (por ejemplo, al no considerar la «inconsistencia de estatus» que se produce cada vez con más fuerza en las migraciones femeninas del «Sur» al «Norte»). Bajo la categoría de «servicios de proximidad» se hace hoy día referencia y principalmente a las actividades del sector terciario dirigidas a la reproducción de la fuerza de trabajo. Son aquéllas tareas relacionadas con el mantenimiento y cuidado de la infraestructura del hogar, mantenimiento y atención de la fuerza de trabajo presente, pasada y futura, la organización y gestión del hogar y la familia, la mediación entre la familia y los servicios públicos y privados existentes, y por último, las tareas de representación conyugal (Torns, 1995a).

Ante esta situación de partida en el mercado de las mujeres inmigrantes hemos podido constatar cómo, con frecuencia, los programas de formación de mujeres inmigrantes no pueden cambiar demasiado las posibilidades de inserción, debido a su situación en el mercado de trabajo; es decir, los procesos de paro y precarización dan poco margen para que se altere el contexto de partida de las mujeres en el mercado. Queda así en la duda hasta qué punto los programas de formación son realmente una herramienta de inserción laboral, o bien son herramientas neutras ante una situación de conflicto, o bien acaban resultando una herramienta parcial vinculada sólo a la integración social y cultural de este colectivo.

Una última dimensión de la eficacia de la acción formativa se establece en términos de estrategia para conseguir la regularización por parte de las mujeres inmigrantes. Esta dimensión es muy difícil de evaluar, no sólo porque no disponemos de datos cuantitativos al respecto, sino porque la constante fluctuación entre el estatus regular e irregular que conlleva la legislación española en materia de extranjería, dificulta enormemente este tipo de análisis. En cualquier caso, sí sabemos que tanto los cursos de formación para mujeres de la asociación SURT como los que ofrece Cáritas, reclutan indistintamente a alumnas regulares e irregulares y les ofrecen asesoramiento y facilidades para tramitar sus "papeles» a lo largo del curso. Desconocemos, en cambio, hasta qué punto las mujeres inmigrantes irregulares (o a punto de perder la condición de regular) conocen esta posibilidad y la utilizan como estrategia.

\section{El doble desdibujamiento de la gestión: modelo horizontal y vertical}

El modelo de políticas públicas de la ciudad de Barcelona respecto a políticas de formación destinadas a la mujer inmigrante se caracteriza por una doble línea de acción: por una parte, una línea horizontal, en la que se insertan las administraciones públicas, ofreciendo una asistencia generalista y donde se pretende la inserción de los distintos colectivos en las líneas de actuación generales del sistema. Por otra, una línea vertical, que atañe a las asociaciones y centros de colaboración, ofreciendo una formación específica y adecuada para los colectivos específicos (gráfico 1). Así, desde las administraciones no se atiende 


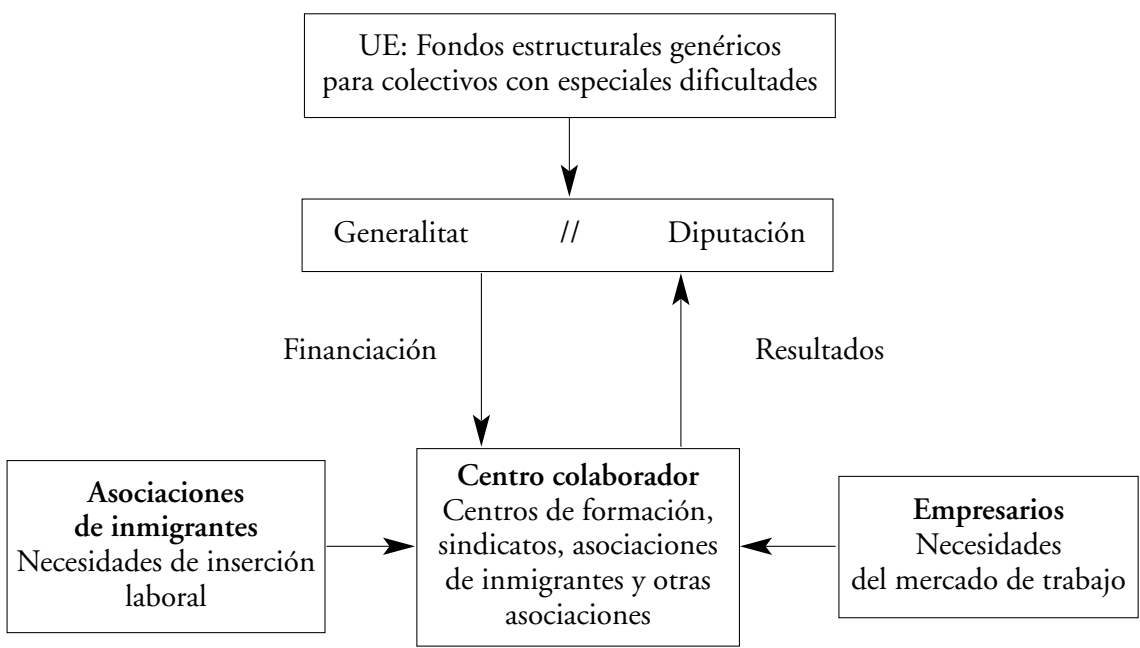

Gráfico 1. La centralidad de los centros colaboradores en el diseño y ejecución de políticas públicas destinadas a la formación de la mujer inmigrante.

Fuente: elaboración propia.

a las especificidades étnicas, puesto que se persigue aplicar un modelo de servicios generalizado, en consonancia con la tradición ya establecida en Cataluña. Las actuaciones específicas se dejan, deliberadamente, en manos del tercer sector. No hay partidas únicas para inmigración, la iniciativa se halla en manos del tercer sector.

El doble desdibujamiento se concreta en un nivel institucional: uno generalista y de programación y otro fuera del ámbito institucional o semiinstitucional, particularista y de gestión directa.

A pesar de todo, no podemos afirmar que exista en la ciudad de Barcelona una política de formación ocupacional específica para mujeres inmigrantes -igual como ocurre con los servicios sociales para inmigrantes-, sino que se procura, en la medida de lo posible, canalizar la formación de este colectivo a través de la oferta de servicios ordinarios. Este propósito se ha manifestado por parte de la totalidad de los actores entrevistados. La formación específica para inmigrantes sólo se defiende como una medida provisional, para aquellos casos en los que se afirma que la inmigrante no puede seguir con normalidad la oferta de cursos general (sea por problemas de idioma o por falta de alfabetización básica).

Los actores con mayor poder político no consideran una prioridad cambiar el modelo de doble dimensión para conseguir que las políticas sean más racionales. Esto queda patente en la clara diferenciación que se hace en el discurso de las administraciones respecto a las características de la población inmigrante y a los mecanismos que pueden mejorar su inserción laboral. Mientras que el discurso de los actores institucionales entrevistados aboga por 
un tratamiento igualitario y generalizado de la población inmigrante, los centros de formación bifurcan su actuación a través de la polarización de los cursos según las características de la población. Esta polarización es uno de los rasgos más acentuados en contraste con los cursos que se destinan a la población autóctona. Así pues, a pesar de la voluntad de no destinar recursos específicos para estos colectivos, la práctica real demuestra que existen dos tipos de ofertas, en función de las características de las mujeres inmigrantes y de sus necesidades formativas, distinguiéndose una población determinada no sólo por las diferencias de etnia y género, sino también por el potencial de formación. Esta polarización se conforma en el establecimiento de dos grupos a dos velocidades: por un lado, las alfabetizadas, con conocimiento del castellano y con experiencia profesional en su país de origen (y consecuentemente con unos recursos actitudinales y conocimiento del entorno concretos), que ejemplifican claramente las peruanas. Por el otro, las semialfabetizadas o analfabetas, con poca experiencia laboral reglada en el país de origen y con cargas familiares, poniéndose frecuentemente como ejemplo a las mujeres marroquíes. El objetivo final es que ambos grupos se acaben adaptando a la oferta formativa general existente. No obstante, dado que las condiciones de partida no son paralelas, las asociaciones toman medidas de discriminación positiva que permitan a las mujeres del segundo grupo obtener unos mínimos conocimientos indispensables (alfabetización y preformación), además de «la autoestima y la autonomía personal» necesarias para poder adquirir posteriormente conocimientos técnicos.

En último término, se persigue tanto su inserción en el mercado de trabajo como su integración en el entorno social en el que viven. No obstante, los cursos analizados suelen partir de una filosofía asistencialista y asimilacionista, de modo que no aprovechan los conocimientos, los hábitos y las aptitudes que estas mujeres adquirieron en sus sociedades de origen. Además, ninguno de los actores analizados diseña los cursos formativos en vistas a un posible enriquecimiento de formación plasmado en un posible retorno, en el caso que se tomen en cuenta potenciales agentes de cambio social en las zonas de origen de las alumnas.

En cuanto al análisis de la población objetivo, otra división a tener presente en estos grupos es la variable regular/irregular. El derecho de acceso a la oferta formativa que existe en la ciudad de Barcelona (tanto si es general como para colectivos con especiales dificultades) se restringe, desde el discurso oficial de las instituciones públicas (Generalitat de Cataluña, Diputació de Barcelona, Ayuntamiento), a inmigrantes en situación regular (con permiso de residencia o con permiso de trabajo). Ahora bien, dado de que se trata de un modelo descentralizado, la práctica cotidiana de los centros colaboradores y de algunas asociaciones permite un cierto margen de discrecionalidad a la hora de reclutar a las candidatas de los cursos. Según las entrevistas, las inmigrantes irregulares no encuentran ninguna dificultad a la hora de ser admitidas en los cursos de formación específicos para mujeres inmigrantes. 


\section{El análisis de la interacción definido por el welfare mix}

Las acciones dependen principalmente de la bondad presupuestaria de entidades extraestatales, nos referimos en concreto a la creciente europeización de los proyectos sociales (especialmente del Fondo Social Europeo) (gráfico 2). Esto ocurre frecuentemente desde una cierta homogeneización de los proyectos en desequilibrio con una heterogeneidad de las realidades. A medida que ha ido aumentando la población inmigrante ha ido a su vez multiplicándose la estructura organizativa de los diferentes actores, así como la dependencia de financiación europea para sus acciones. Este nuevo sistema de dependencia europea implica la carencia de una autonomía financiera por parte de las administraciones a todos los niveles, desde el estatal hasta el local.

El organigrama de las políticas de formación para mujeres inmigrantes se corresponde, prácticamente, con el modelo de formación ocupacional descentralizado que sigue la Generalitat de Catalunya desde 1989. El actor central en el diseño y ejecución de las acciones destinadas a mujeres inmigrantes son los centros colaboradores (gráfico 3), los cuales pueden formar parte de la propia Administración, del mercado o bien del tercer sector. El centro colaborador realiza la prospección de mercado; analiza cuáles son las necesidades y los beneficiarios potenciales; elige a sus formadores y los contenidos de los cursos y se encarga de elaborar un plan de inserción. Al Departament de Treball le

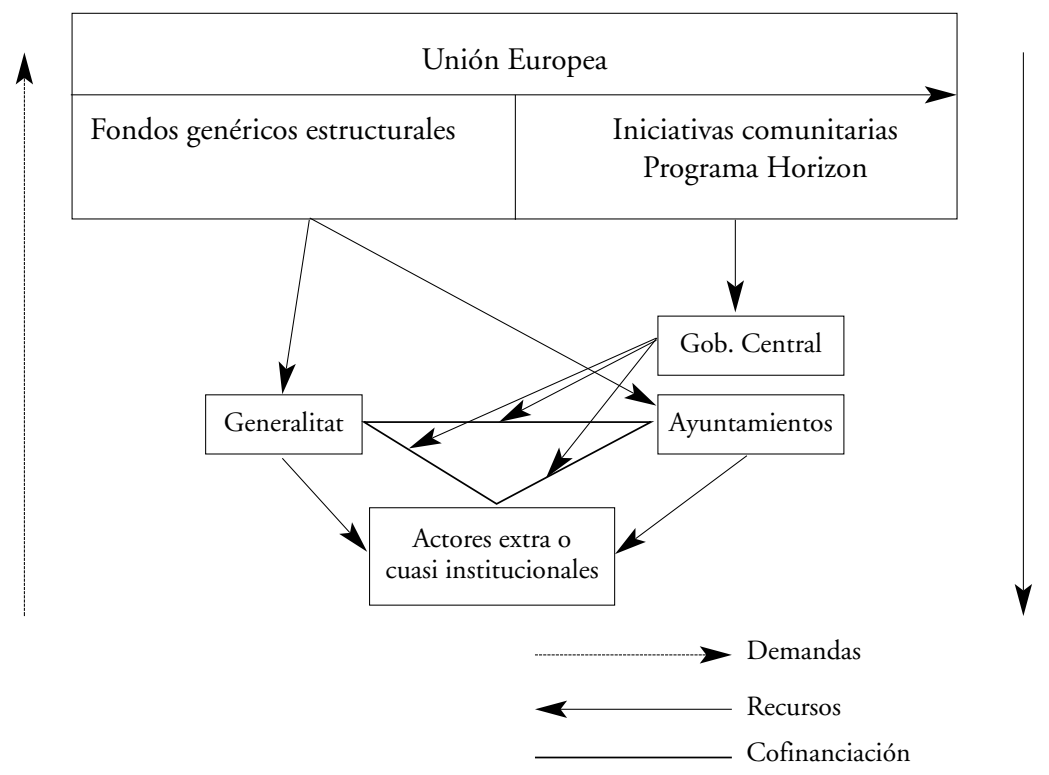

Gráfico 2. Líneas de financiación de las políticas públicas de inserción en el mercado de trabajo de mujeres inmigrantes en Barcelona.

Fuente: elaboración propia. 


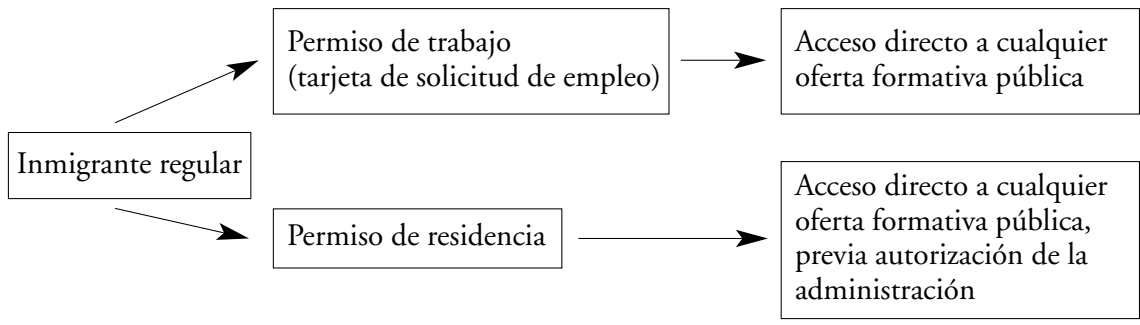

Inmigrante irregular

Formalmente, no puede acceder a la oferta formativa pública. Su acceso queda sujeto a la postura que adopten cada una de las entidades formativas

Gráfico 3. Relación del tipo de inmigrante con el acceso a la formación.

Fuente: elaboración propia.

corresponde la tarea de selección y aprobación de los distintos proyectos que presentan los centros colaboradores y de la evaluación ex-post de los resultados obtenidos.

Pese a la concurrencia de competencias por parte de las distintas administraciones, es difícil observar en nuestro ámbito de estudio un solapamiento concreto de acciones, sea en el espacio o en el tiempo. Esto es debido, por una parte, a las restricciones presupuestarias con las que cuentan los organismos públicos y, por otra parte, al hecho de que las acciones se llevan en la práctica desde el ámbito municipal (donde se suman recursos más que ofertas concurrentes).

Por parte de la Administración pública no existe un previo planteamiento global de las acciones que contemple la evaluación de las necesidades de las mujeres inmigrantes a las que deba responderse, ni siquiera a largo plazo. Tampoco hay ninguna concepción ni diseño de un marco global que coordine el conjunto de iniciativas. Todo está en función de las iniciativas descentralizadas. Si bien este modelo ofrece algunas ventajas, en tanto que son los ayuntamientos y las asociaciones las que mejor conocen las necesidades de estos colectivos y su evolución, la carencia de una línea de actuación coordinada corre el riesgo de excluir a ciertos grupos desaventajados dentro del colectivo de mujeres inmigrantes.

La formación ocupacional en general y, para el caso que nos ocupa, la formación ocupacional para mujeres inmigrantes extranjeras en la ciudad de Barcelona, obedece a un modelo de welfare mix, basado en una orientación pluralista del bienestar en la protección social. En nuestro caso participan distintos actores: la Administración autonómica (Direcció General d'Ocupació 
i Institut Català d'Assistència Social), la Administración local (ayuntamientos y Diputación de Barcelona) y el tercer sector, que engloba tantos los actores cuasi institucionales (sindicatos, Cáritas), como los extrainstitucionales (asociaciones de inmigrantes, asociaciones de mujeres, asociaciones generalistas...). Las organizaciones patronales quedan totalmente al margen, así como la iniciativa meramente privada, dadas las dificultades de inserción laboral de este colectivo.

La opción de welfare mix en la gestión y administración de servicios formativos dirigidos a la población inmigrante puede considerarse como paradigmática de este modelo, al haberse institucionalizado el papel de estos actores en el proceso de implementación de las políticas de formación para mujeres inmigrantes. Este proceso obedece, según su incidencia explicativa, a las siguientes razones:

- El objetivo de las administraciones públicas de delegar la ejecución de dichas políticas sin ninguna regulación formal al respecto se ve satisfecho. Éstas limitan su conjunto de opciones en materia de realización de las tareas de ejecución, a la vez que controlan el elemento principal condicionante de las estrategias de todos los actores: la financiación.

- La mayoría de los centros de formación no discuten este marco de interdependencia. En el caso de los actores que abogan por una mayor regulación, como son los sindicatos, todo apunta a que lo consideran inviable: las preferencias inmediatas de dirigirse a esta población y de satisfacer sus demandas posponen las preferencias a largo plazo de estos actores.

- Por último se constata en los últimos años un incremento del número de actores del tercer sector en cuanto a iniciativas relacionadas con la inmigración.

\section{El resultado de las políticas: una solución con dos caras}

Finalmente, debemos apuntar que las hipótesis aquí expuestas deberían de momento concebirse sólo como hipótesis de trabajo; la razón no es otra que la insuficiencia de datos utilizados, muchas veces a causa de su propia inexistencia. Los datos todavía son reducidos, incluso si sólo queremos aplicarlos al caso de Barcelona.

Nos referimos al doble juego de las acciones, por una parte, como medida de formación y, por otra, como medida de socialización. En cuanto a la formación se destaca la reproducción de los trabajos tradicionalmente femeninos, que son a su vez nuevas necesidades de la sociedad pese al cambio de estatus y de régimen laboral en ciertos casos (por ejemplo, los trabajos de geriatría y auxiliar de cocina). En cuanto a la socialización, las acciones se dirigen hacia un proyecto general de integración social muy definido según los actores entrevistados: hablar la lengua y adquirir «autonomía personal», entre otras características. A corto y largo plazo, los resultados de las políticas de formación regulan y potencian los rasgos estructurales de los segmentos del mercado labo- 
ral que caracterizan a las mujeres inmigrantes: canalización de fuerza de trabajo inmigrante femenina en el segmento de mercado secundario, reproducción de las estructuras profesionales típicamente femeninas y potenciación de las actitudes que decantan la lucha por el control del proceso de trabajo a favor de los empresarios. Estos resultados también están muy vinculados a las políticas de contingentes impuestas por el gobierno español.

Las funciones expresadas por los actores también muestran dos caras: una función manifiesta de integración, sobre todo por parte de SURT y de Cáritas, y una función muchas veces latente que es la asimilación, sobre todo por parte de las diferentes administraciones.

El proceso de interacción entre los actores de las políticas presenta un carácter reproductivo, esto es, los efectos de sus políticas parecen no alterar las características del entorno estructuradas en la relación entre formación y empleo, ni varían los rasgos de la interdependencia entre los actores de las políticas públicas. De esta manera, la configuración ex-ante y ex-post de las políticas públicas presenta unos objetivos, unas estrategias y unos resultados similares en todos los periodos del tiempo.

\section{Bibliografía}

ABRAHAMSON, P. (1995). «Regímenes europeos del bienestar y políticas sociales europeas: ¿convergencia de solidaridades?». En SARASA, S.; MORENO, L. (comp.). El Estado del Bienestar en la Europa del Sur. Madrid: CSIC.

Ajuntament de Barcelona (1997). Pla intercultural. Barcelona: Ajuntament de Barcelona.

BECKMAN, N. (1992). «Policy analysis in government: Alternatives to muddling thought». Public Administration Review, 37.

CASEY, J. (1997). El papel de las organizaciones no gubernamentales en la elaboración de las políticas públicas. Ajuntament de Barcelona. Dossiers Barcelona Associacions, núm. 20.

Generalitat de Catalunya (1994). Pla Interdepertamental d'Immigració. Barcelona: Generalitat de Catalunya.

GinTIS, H. (1983). «La naturaleza del intercambio laboral y la teoría de la producción capitalista». En TOHARÍA, L. (comp.). El mercado de trabajo: teorías y aplicaciones. Madrid: Alianza.

Juliano, D. (1998). Las que saben. Subculturas de mujeres. Madrid: Horas y horas. MENY; THOENIG, J.C. (1992). Las politicas públicas. Barcelona.

Oso, L. (1998). La migración a España de las mujeres jefas de hogar. Madrid: Instituto de la Mujer.

PiORE, M. (1983). «Notas para una teoría de la estratificación del mercado de trabajo». En TOHARIA, L. (comp.). El mercado de trabajo: teorías y aplicaciones. Alianza: Madrid.

RibAS, N. (1998). «Política social: género e inmigración». Revista Ofrim de la Comunidad Autónoma de Madrid.

SÁEZ, F. (1997). «Políticas de mercado de trabajo en Europa y en España». Papeles de Economía española, 72: 309-325.

SOlÉ, C. (1994). Mujer Inmigrante. Madrid: Instituto de la Mujer. 
Subirats, J. (1992). El análisis de políticas públicas. Madrid: INAP.

TORns, T. (1995a). "Mercado de trabajo y desigualdades de género». Cuadernos de relaciones laborales, 6 . Madrid.

- (1995b). "Los nuevos empleos, cualificación y valoración». Comunicación presentada en el Séminaire IRIS: Une vision plus large. Bruselas, junio de 1995.

WalBy, S. (1986). Patriarchy at work. Patriarchal and capitalist relations in employment. Minneapolis: University of Minessota Press. 